\title{
Mitigating noise effects in volatile nano-metal oxide neural detector
}

\author{
Isha Gupta*, Alexantrou Serb*, Ali Khiat*, Themistoklis Prodromakis* \\ ${ }^{*}$ Department of Electronics and Computer Science, University of Southampton, SO17 1BJ, UK \\ Corresponding author email: I.Gupta@soton.ac.uk
}

\begin{abstract}
The sensitivity of a recently proposed spike detector exploiting the volatile properties of memristive device is optimised. A $200 \mathrm{~nm} \times 200 \mathrm{~nm} T i O_{x}$ memristive device in volatile region is biased with sub-threshold, unipolar and bipolar neural events. The input neural signal is pre-processed using different amplification settings. The resistive state response of the test device in response to the input events is analysed and it is found that inclusion of events in positive polarity leads to subsequent increase in the number of false events when benchmarked against state-of-the-art spike detector (template matching system). The performance of the system is thereafter optimised by determining optimum amplification settings and employing an offset such that positive polarity events in the input signal are minimised.
\end{abstract}

Index Terms-Memristors, RRAM, integrating sensor, volatility, noise, template matching system

\section{INTRODUCTION}

Advances in neuroscience research and microelectronic devices have led to substantial progress in neural processing microsystems bearing great potential for neuroprosthetic applications [1], [2]. Present date neural recording techniques [3] are capable of recording electrophysiological activity from large number of neurons in-vitro and in-vivo [4] leading to creation of big neural data $(\mathrm{Gb} / \mathrm{s})$ [5]. Further advances in development of neural interfaces is constrained by computational power required to process ever increasing volume of neural signals on-node in real-time [6], bandwidth [7] and scalability.

Recently, we proposed a new spike-detection approach exploiting the volatile properties of emerging metal-oxide resistive switching memory devices [8], commonly known as 'memristors' [9]. When operated in volatile region, the devices tend to undergo metastable memory state transitions above an inherent threshold following which they inherently relax to their initial resistive state (RS) region [10]. Using nano devices, we demonstrated that the spiking events can be encoded into transient RS changes of device-under-test (DUT) reducing the power dissipated to less than $100 \mathrm{nW}$ per channel [8]. However, the study did not examine the effect of subthreshold events and operational parameters such as signal amplification and offset on the response of memristive devices in volatile region.

We acknowledge the financial support of FP7 RAMP and EPSRC EP/K017829/1. We thank Prof Stefano Vassanelli at University of Padova, Italy and Prof Ralf Zeitler at Max Plank Insitute of Intelligent Systems, Germany for providing us the neural recordings and relevant guidance through the experiment. Experimental procedures invovlving the use of animals were approved within the RAMP projects by Ethics Committee of the University of Padova and the Italian Ministry of Health (authorisation.447/2015-PR). Al the experiments were conducted in accordance with the approved guidelines. (a) Electrical Characterisation of Memristive Devices

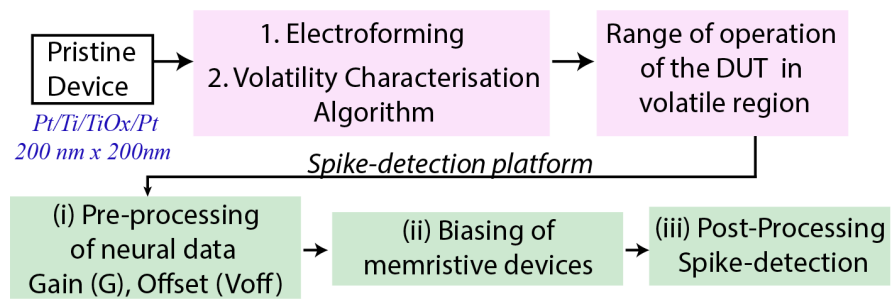

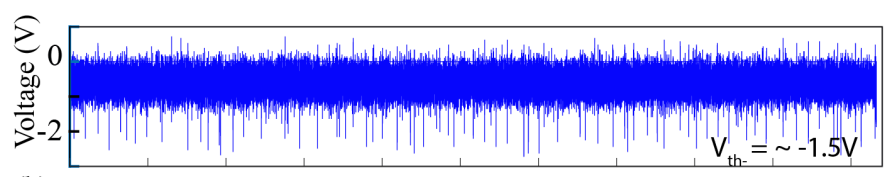

(b)

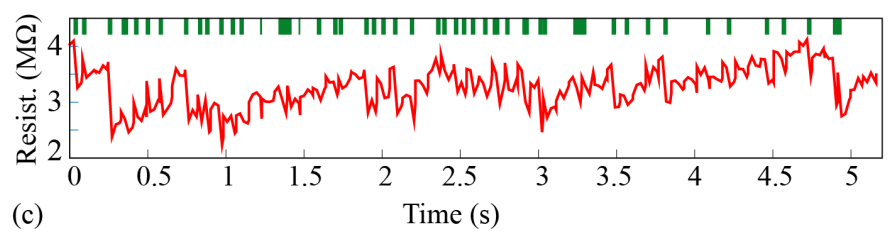

Fig. 1. (a) Flow chart of electrical characterisation of memristive devices in the volatile region and the spike-detection platform. (b) Neural recording used for the experiment. (c) Resistive state evolution of the DUT in response to the neural recording. The green band indicates the number of spikes detected by the our platform.

In this work, we analyse the effects of sub-threshold, unipolar and bipolar neural events on a $\mathrm{TiO}_{x}$ nano memristive device. The spike-detection performance of the devices is estimated and benchmarked against established state-of-the-art template matching system (TMS) [11]. Important observations are made and the operational parameters are then optimised to demonstrate enhanced sensitivity of the devices to biased spiking events.

\section{System IMPLEMENTATION}

A. Devices, Hardware Infrastructure and Electrical Characterisation of volatile memristive devices

Figure. 1a illustrates the methodology and sequence in which the experiments were carried out. For these experiments, solid-state $200 \mathrm{~nm} \times 200 \mathrm{~nm}$ nano devices with stack structure of $\mathrm{Ti} / \mathrm{Pt} / \mathrm{TiO}_{x} / \mathrm{TiN}$ were employed [12]. The electrical characterisation was carried out using custom made inhouse fabricated hardware capable of addressing up to 32 devices in stand-alone configuration. In this work devices were accessed directly on-wafer via a probe card [13]. The 
electrical characterisation of devices was carried out in two stages: (a) the pristine devices were electroformed using pulses of positive polarity in the range of $+4 \mathrm{~V}-+6 \mathrm{~V}$ [8] and (b) the devices are biased with volatility characterisation algorithm to determine the safe volatile region of operation of devices (i.e. up to approximately $-4 \mathrm{~V}$ ) [14]. Notably, the devices in volatile region operate in resistive state range of $300 \mathrm{k} \Omega-3 \mathrm{M} \Omega$ and the inherent threshold $\left(V_{t h-}\right)$ of the devices are in the range of $\approx-0.5 \mathrm{~V}--2.5 \mathrm{~V}[8]$

\section{B. Extracellular neural recordings}

The neural recording used in this work was recorded from slices of dissected mid-peripheral rabbit ganglion cells using an external Multi Transistor Array (MEA) based Complementary Metal-Oxide Semiconductor (CMOS) front-end system [15]. The output neural recordings are voltage-time signals in the range of $\pm 0.5 \mathrm{~V}$ recorded at a sampling rate of $12.2 \mathrm{kHz}$. Each neural recording in this work contains $\approx 63 \mathrm{k}$ samples lasting for $\approx 5.2 \mathrm{~s}$ (as shown in Fig. 1b) [16].

\section{Spike-detection platform and signal processing}

In the spike-detection platform, the extracellular recordings are pre-processed and suitably amplified using a gain $(G)$ and offset $\left(V_{o f f}\right)$ stage. Next, the DUT is biased with the processed neural recording using the hardware infrastructure and the RS transitions of the device are recorded periodically. The compressed RS readings are thereafter post-processed to distinguish significant RS changes from the insignificant ones. Figure 1b,c illustrates an example of the volatile RS response of the DUT in response to the biased neural recording amplified using a $\mathrm{G}$ and $V_{\text {off }}$ value. The initial RS of the DUT is $\approx 4 \mathrm{M} \Omega$. With respect to supra-threshold event the device exhibits a metastable transition to a low $\mathrm{RS}$ of $\approx 3.2 \mathrm{M} \Omega$ following which the devices spontaneously relaxes back.

Importantly, in this work we follow a standard schematic and the neural signal is fed to the DUT in batches of 1000 data points [16]. Per batch five RS measurements are recorded $($ Compression rate $=1000 / 5=200)$. In every batch the RS of the DUT is recorded in the beginning of each batch and then after every 300 data points giving four consecutive RS measurements (i.e. four bins). Thereafter one reading is taken at the end of each batch and the beginning of next batch with no neural data point in between (i.e one bin). The former RS measurements are used for estimation of normalised RS changes in each bin $\left(\Delta \mathrm{R} / R_{0}\right)$ and the latter gives the estimation of noise in the system. Collection of the noise measurements make a noise band. RS change in each bin $\left(\Delta \mathrm{R} / R_{0}\right)$ is assigned to the highest voltage magnitude in each bin. For the noise band limits, since the DUT relaxes in positive polarity, all the noise measurements in this polarity are discarded and only measurements in negative polarity are used [8]. The significant RS changes in comparison to the noise band gives an estimation of the number of spikes detected. All the RS changes outside the noise band are estimated as detected spikes.

The number of spikes detected are benchmarked against state-of-the-art TMS. For the purpose of quantification and
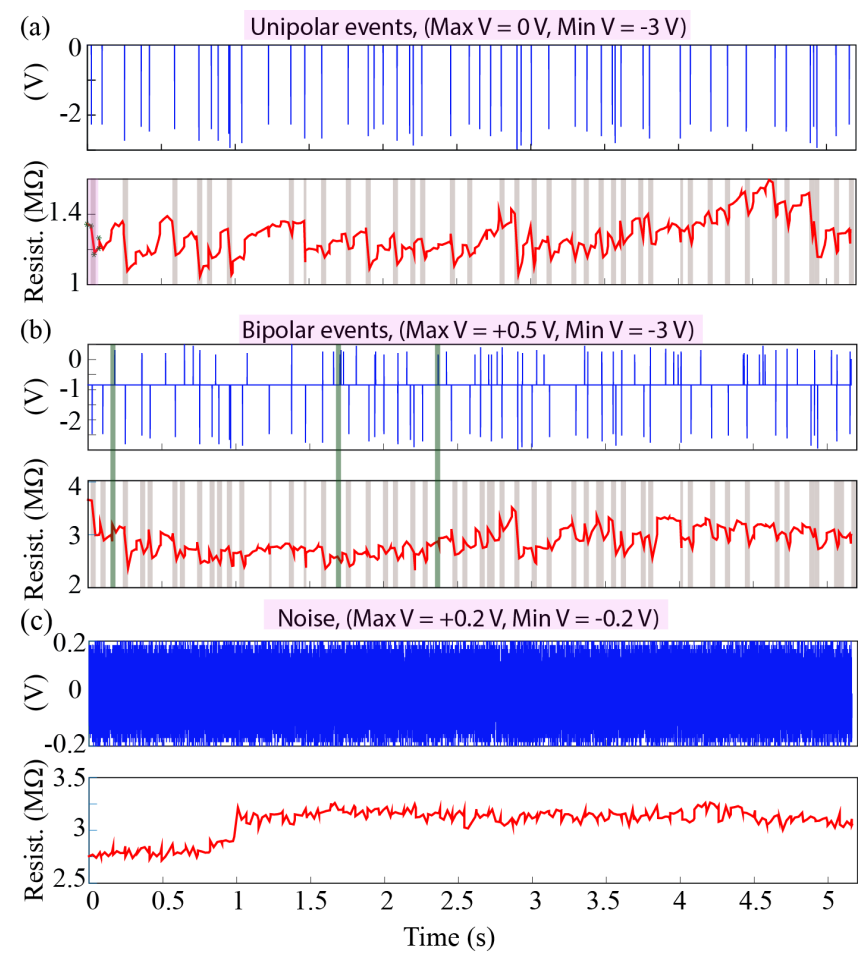

Fig. 2. Analysing the effect of events on nano volatile memristive device. (a), (b) (c) (Upper panel's) Biasing a device-under-test (DUT) with unipolar, bipolar and noise events, pre-processed using different settings. (a), (b), (c) (Lower panel's) Response of the DUT in response to the biased events. The grey bands indicates the spikes detected by our platform. The green bands indicates few instances of spikes detected due to positive polarity events.

TABLE I

COMPARISON OF QUANTIFICATION PARAMETERS FOR THE UNIPOLAR AND BIPOLAR EVENTS IN 2A AND B. VMS: SPIKES DETECTED BY OUR PLATFORM, TMS: SPIKES DETECTED BY TEMPLATE MATCHING SYSTEM, True positives (TP), False Positives (FP), True Negatives (TN) AND FALSE NEGATIVES (FN)

$\begin{array}{cccccccccc} & \begin{array}{c}\text { Max/Min } \\ (\mathrm{V})\end{array} & \mathrm{VMS} & \mathrm{TMS} & \mathrm{TP} & \mathrm{FP} & \mathrm{TN} & \mathrm{FN} & \begin{array}{c}\text { RTP } \\ (\%)\end{array} & \begin{array}{c}\text { RFP } \\ (\%)\end{array} \\ 1 . & 0 /-3 & 37 & 78 & 35 & 2 & 173 & 43 & 44.8 & 1.14 \\ \text { 2. } & +0.5 /-3 & 57 & 78 & 46 & 11 & 164 & 32 & 58.9 & 6.28\end{array}$

understanding the efficiency of the neural detector, the rate of TP (RTP) and FP (RFP) are calculated [17].

\section{RESUlTS AND Discussion}

\section{A. Effects of unipolar and bipolar neural events}

For this experiment raw neural recording i.e. prior to preprocessing was modified. To generate events in one polarity as shown in Fig. 2a, the threshold was set to $-0.5 \mathrm{~V}$ and all the events above were discarded to study only the effects of spiking events in one polarity. The remaining events were then amplified to $[0,-3 \mathrm{~V}]$ range. Similarly for Fig. $2 \mathrm{~b}$, two threshold's were set at $+0.3 \mathrm{~V}$ and $-0.5 \mathrm{~V}$ and the remaining events above and below respectively were amplified to $[+0.5 \mathrm{~V}$, $-3 \mathrm{~V}]$ range to the study the relation of spiking events in one polarity to events in different polarity. The RS transitions of a DUT in response to the pre-processed signals are illustrated in Fig. $2 \mathrm{a}$ and $\mathrm{b}$ respectively. The grey band are spikes detected by our platform. 


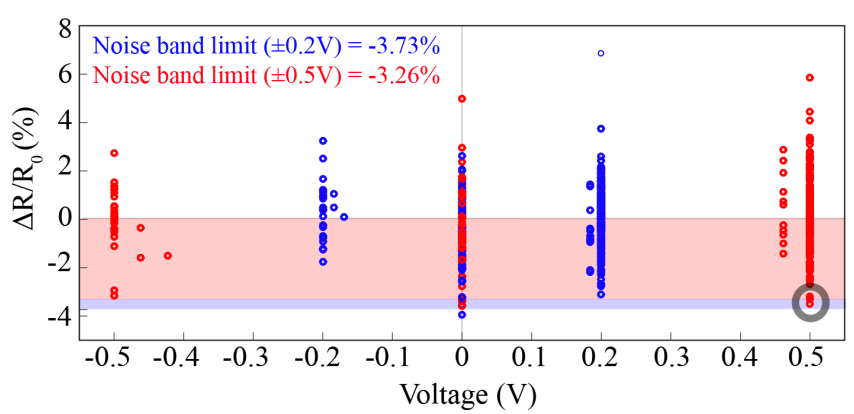

Fig. 3. Comparison of noise band diagrams i.e. $\Delta \mathrm{R} / R_{0}$ vs voltage plots for sub-threshold events. The blue and red colour illustrates the response of the DUT in response to the neural events in Fig. $2 \mathrm{c}$ when mapped to approximately $\pm 0.2 \mathrm{~V}$ and $\pm 0.5 \mathrm{~V}$ respectively.

The effect of positive polarity events for instance at $\approx 0.3 \mathrm{~s}$, $1.6 \mathrm{~s}$ and $2.4 \mathrm{~s}$ as illustrated by green bands in Fig. $2 \mathrm{~b}$ can be noted. The quantification parameters are presented in Table. I and it can be noted that the inclusion of positive polarity events increases the RTP from $44.8 \%$ to $58.9 \%$. However, the RFP correspondingly increases from $1.14 \%$ to $6.28 \%$. Moreover, in our platform the RS change within a bin (300 data points) is attributed to the strongest pulse, therefore at number of bipolar instances such as $0.4 \mathrm{~s}, 1.5 \mathrm{~s}, 2 \mathrm{~s}, 3.4 \mathrm{~s}, 4.4 \mathrm{~s}$ etc the change in $\mathrm{RS}$ is assigned to negative polarity events.

\section{B. Effect of sub-threshold events}

The $V_{t h-}$ of the employed devices are in the range of $\approx 0.5 \mathrm{~V}$ to $2.5 \mathrm{~V}$. To understand the effect of sub-threshold events on the volatile behavior of the devices, the following experiment was carried out. Similarly to Fig. 2a, two thresholds were set to $+0.3 \mathrm{~V}$ and $-0.5 \mathrm{~V}$ and the activity within was amplified to $[+0.2,-0.2] \mathrm{V}$ range deliberately excluding the spikes, as shown in Fig. 2c. Notably, a drift in the RS of the DUT from $\approx 2.8 \Omega$ to $\approx 3.2 \mathrm{M} \Omega$ in response to the sub-thresholds can be noted.

For better understanding, the normalised changes in the RS of the DUT in each bin $\left(\Delta \mathrm{R} / R_{0}\right)$ is plotted as a function of the highest voltage magnitude in each bin (as shown in blue colour, Fig. 3). The noise measurements are illustrated on $\mathrm{x}=0$, the measurements in positive polarity are discarded and the noise band limit is set using $2 \sigma$ method using negative noise measurements (optimised for our system) ${ }^{1}$. The noise band limit is estimated to be $-3.73 \%$. It can be observed that all RS changes at $\approx \pm 0.2 \mathrm{~V}$ fall within the estimated noise band limits. Since, we account for normalised changes in RS in each bin, slight drift in the RS of the DUT is naturally filtered off and therefore these voltages doesn't affect the output of the proposed neural detector.

However, on amplifying the neural events in Fig. $2 \mathrm{c}$ to $\pm 0.5 \mathrm{~V}, \Delta \mathrm{R} / R_{0}$ vs $\mathrm{V}$ plot in red colour in Fig. 3 is obtained. The noise band limit is estimated to be $-3.26 \%$ and the RS changes in black circle at $+0.5 \mathrm{~V}$ fall outside the noise band

${ }^{1}$ It should be noted that resistive state changes in positive polarity are discarded. Since, the devices relaxes back in positive polarity following a resistive state transition, inclusion of noise events in positive polarity doesn't indicate fair estimation of the noise band limits. thus registered as spikes confirming the effect of positive polarity events. Following these observations positive polarity events were modulated using an optimum offset value such that strength of events in positive polarity were minimised, which also justifies the importance of this key operational parameter.

\section{Optimum settings for volatile devices}

Considering the observations made in Fig. 2 and Fig. 3, the operational parameters for pre-processing the neural signal were reconsidered and optimised. The same neural recording (as shown in Fig. 1b) was amplified using different gain values whilst the offset value was kept constant. Figure. 4 a, b and $c$ illustrates the $\left(\Delta \mathrm{R} / R_{0}\right)$ vs $\mathrm{V}$ plots for the response of a DUT when biased with same neural recording with $G$ value set as 2.6, 3.2 and 3.6 respectively. The offset was kept constant at $-0.6 \mathrm{~V}$. The number of spikes detected by our platform was benchmarked against the TMS and the quantification parameters are demonstrated in Table. II

Visually in Fig. 4a,b and c, the grey area indicates the noise measurements that have been discarded. The horizontal blue, red and green bar indicates the noise band with black dashed line indicating the noise band limit. Everything outside the noise band in the negative quadrant indicates the number of detected spikes. This can be also seen in histogram plots presented in Fig. 4c, d and e, where the yellow bands indicate the spikes detected by our system. It was observed that as the gain was increased from 2.6 to 3.2 , the rate of TP almost doubled i.e from $29.48 \%$ to $60.25 \%$. At the same time the corresponding increase in the rate of FP was from $2.8 \%$ to $5.14 \%$ (see Table. II). More clearly, on comparing Fig. 4 a,b the pink quadrants in the latter indicates a significant increase in the detected spikes thus indicating the positive effects of increase in gain. The noise band limit in both the cases is $\approx$ $-4.5 \%$. Similar observations were made for $\mathrm{G}=2.8$, where the rate of TP was found to be $38.48 \%$.

Interestingly, as the gain was further increased from 3.2 to 3.6, the rate of TP remained approximately the same, however, the rate of FP almost doubled to $9.71 \%$ where the noise band limit was estimated to be $-4.2 \%$ (see Table. II). Gain (3.6) and $V_{\text {off }}(-0.6 \mathrm{~V})$ for this DUT in essence illustrates the optimum point of operation. This can be generalised to an understanding where most significant supra-threshold events are above the inherent threshold of the device and insignificant events/noise are below the threshold. An increase in gain beyond an optimum point leads to inclusion of insignificant amplified noise events above the threshold of the DUT and thus effects the sensitivity of the neural detector by increasing the FP.

\section{Discussion}

The presented optimised and quantified results are from devices in nano dimensions (200 nm x $200 \mathrm{~nm})$. We obtain much higher rate of $\mathrm{TP}$ and $\mathrm{FP}$ routinely with higher dimension devices of $60 \mu \mathrm{m} \times 60 \mu \mathrm{m}(\approx \geq 75 \%)$ [8]. Exploring and engineering other stack configurations may be other possible route for optimising the performance of the devices. Importantly, the number of spikes detected are benchmarked assuming 

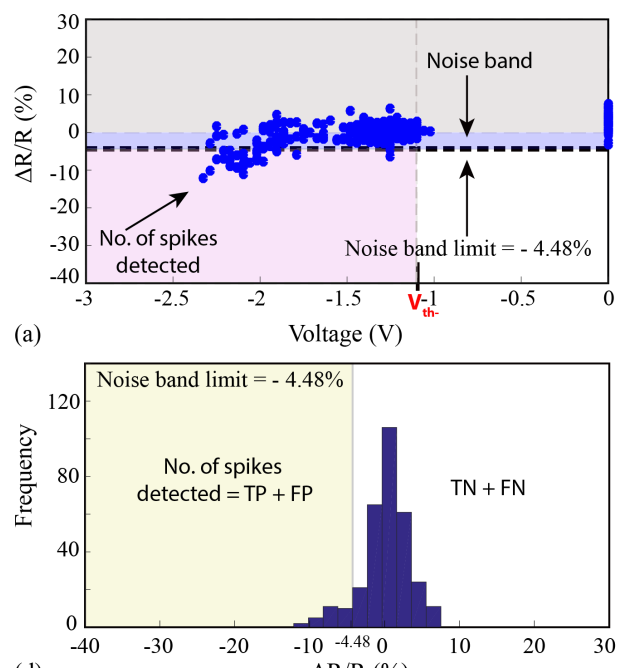

(d)

$\Delta \mathrm{R} / \mathrm{R}(\%)$

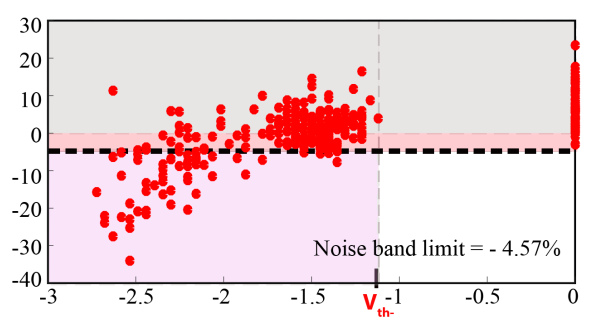

(b)

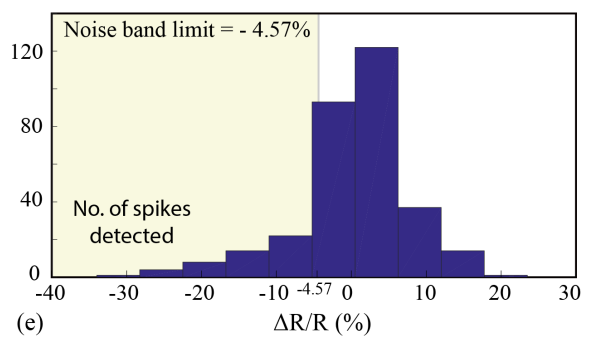

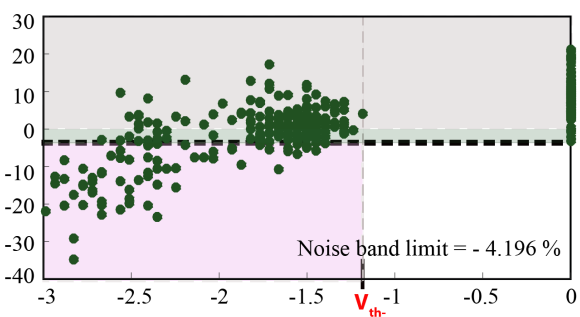

(c)

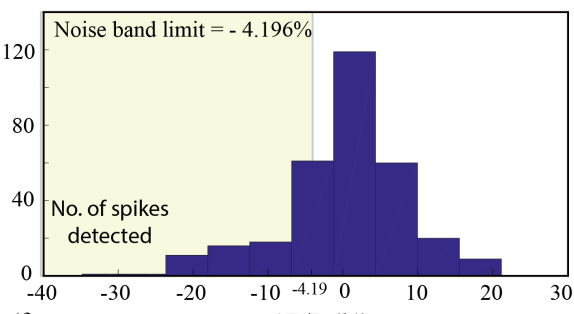

(f) $\quad \Delta \mathrm{R} / \mathrm{R}(\%)$

Fig. 4. A single DUT was biased with the neural recording in Fig. 1b. (a), (b), (c) The plots for $\left(\Delta \mathrm{R} / R_{0}\right)$ with respect to highest voltage magnitude in each bin for gain equal to $2.6,3.2$ and 3.6 respectively. The offset was kept constant at $-0.6 \mathrm{~V}$. (d), (e), (f) Corresponding histograms for $\left(\Delta \mathrm{R} / R_{0}\right)$ for the three cases respectively. The yellow bands indicate the number of spikes detected. $V_{t h}$ - indicates the inherent threshold voltage of the DUT $(\approx-1.2 \mathrm{~V})$.

TABLE II

COMPARISON OF DIFFERENT GAINS WITH CONSTANT OFFSET VALUES FOR A SINGLE NEURAL RECORDING

$\begin{array}{cccccccccccccc} & \text { Gain } & \text { Offset } & \text { VMS } & \text { TMS } & \text { TP } & \text { FP } & \text { TN } & \text { FN } & \begin{array}{c}\text { Rate of } \\ \text { TP }(\%)\end{array} & \begin{array}{c}\text { Rate of } \\ \text { FP }(\%)\end{array} & \begin{array}{c}\text { Noise } \\ \text { Max }(\%)\end{array} & \begin{array}{c}\text { Noise } \\ \text { Min }(\%)\end{array} & \begin{array}{c}\text { Noise band } \\ \text { limit }(\%)\end{array} \\ 1 . & 2.6 & -0.6 & 28 & 78 & 23 & 5 & 170 & 55 & 29.48 & 2.8 & +7.52 & -3.15 & -4.48 \\ \text { 3. } & 3.2 & -0.6 & 56 & 78 & 47 & 9 & 166 & 31 & 60.25 & 5.14 & +23.51 & -2.99 & -4.57 \\ 4 . & 3.6 & -0.6 & 63 & 78 & 46 & 17 & 158 & 32 & 58.9 & 9.71 & +21.15 & -3.18 & -4.196\end{array}$

the template matching system to be a perfect spike-detector but in reality this is not the case [16]. In future, we further plan to confirm the performance of the devices with respect to artificial simulated data where the ground truth is already known. Finally, there may be other ways of setting the noise band limits such as noise max and noise min (also indicated in Table. II), however, the chosen $2 \sigma$ method has been optimised for our devices and system.

\section{CONCLUSION}

We have shown the capability of $\mathrm{TiO}_{x}$ solid-state devices to encode spiking events in the metastable resistive state transitions. The volatility property of memristive devices offers advantages in terms of reduced power dissipation i.e. sub 100 $\mathrm{nW}$ by operating the devices in a very high resistive state region and also offers huge potential for scalability. We presented experimental results from $200 \mathrm{~nm}$ x $200 \mathrm{~nm}$ nano $\mathrm{TiO}_{x}$ memristive devices. The effects of sub-threshold, bipolar and unipolar neural events on the volatile behavior of the device are studied. By mitigating the noise effects and setting an optimum point of key operational parameters, the performance of the neural detector is enhanced.

\section{REFERENCES}

[1] X. Navarro et al., "A Critical Review of Interfaces with the Peripheral Nervous System for the Control of Neuroprotheses and Hybrid Bionic Systems," Jour. of Peri. Nervous System, vol. 10, pp. 229-258, 2005.

[2] S. Vassanelli et al., "Trends and Challenges in Neuroengineering: Toward Intelligent Neuroprostheses through Brain-Brain Inspired Systems Communication," Front. in Neuro., vol. 10, no. September, p. 438, 2016.
[3] F. Franke et al., "High-density microelectrode array recordings and realtime spike sorting for closed-loop experiments : an emerging technology to study neural plasticity," vol. 6, no. December, pp. 1-7, 2012.

[4] Z. Berényi, Antal, Somogyvári et al., "Large-scale, high-density (up to 512 channels) recording of local circuits in behaving animals." Journal of neurophysiology, vol. 111, no. 5, pp. 1132-49, mar 2014.

[5] K. P. Stevenson, Ian HKording, "How advances in neural recording affect data analysis." Nature neuroscience, vol. 14, no. 2, pp. 139-42, feb 2011.

[6] S. E. Paraskevopoulou et al., "A sub-1uW neural spike-peak detection and spike-count rate encoding circuit," in 2011 IEEE Biomedical Circuits and Systems Conference (BioCAS). IEEE, nov 2011, pp. 29-32.

[7] A. Eftekhar et al., "Towards a Next Generation Neural Interface : Optimizing Power, Bandwidth and Data Quality," pp. 122-125, 2010.

[8] I. Gupta et al., "Sub $100 \mathrm{nW}$ volatile nano metal-oxide memristor as synaptic-like encoder of neuronal spikes," arXiv.

[9] A. Jeong et al, "Emerging memories: resistive switching mechanisms and current status," Rep. on Prog. in Physics, vol. 75, no. 7, jul 2012.

[10] I. Valov et al., "Nanobatteries in redox-based resistive switches require extension of memristor theory." Nat. Comm., vol. 4, p. 1771, 2013.

[11] R. Lambacher, A., Vitzthum, V., Zeitler et al., "Identifying firing mammalian neurons in networks with high-resolution multi-transistor array (MTA)," Applied Physics A, vol. 102, no. 1, pp. 1-11, sep 2010.

[12] A. Khiat et al., "High Density Crossbar Arrays with Sub- 15 nm Single Cells via Liftoff Process Only." Scientific reports, vol. 6, p. 32614, 2016.

[13] A. Serb et al., "Live demonstration : A versatile, low-cost platform for testing large ReRAM cross-bar arrays ." IEEE International Symposium on Circuits and Systems, vol. 9, no. 5, p. 4799, 2014.

[14] I. Gupta et al., "Volatility Characterisation for RRAM devices," IEEE Electron Device Letters, 2016.

[15] C. Eversmann, Björn, Jenkner, Martin, Hofmann, Franz, Paulus et al., "A 128x128 CMOS Biosensor Array for Extracellular Recording of Neural Activity," IEEE Journal of Solid-State Circuits, vol. 38, no. 12, pp. 2306-2317, 2003.

[16] I. Gupta et al., "Real-time encoding and compression of neuronal spikes by metal-oxide memristors," Nat. Comm., vol. 7, pp. 1-16, 2016.

[17] I. Gupta et al, "Improving detection accuracy of memristor-based biosignal sensing platform," IEEE Transactions on Biomedical Circuits and Systems, vol. PP, no. 99, pp. 1-9, 2016. 